stantive debate, and in its willingness to abandon ideas that have been found wanting." But as good as this sounds, it fails to acknowledge the central importance to science of a theoretical framework. Of all the many claims of the paranormal that Sagan catalogues, only perpetual motion is rejected on theoretical grounds; all the others are rejected for lack of evidence. Thus, both in theory and practice, Sagan takes an essentially empirical-inductivist view of science.

This leaves him combating pseudoscience with one arm tied behind his back, for without a positive theoretical reason ("We know things don't work that way!") the mere lack of evidence is a weak argument against belief. No matter how many alien abduction scenarios and UFO sightings are explained away, one can never answer the reasonable question of one of his Parade readers: "A hallucination might account for $99 \%$ [of reported alien abductions], but can it ever account for $100 \%$ ?"

Since at least the time of Archimedes, science has deduced results from very general principles, which are not themselves capable of direct verification. The Greeks, for example, deduced the law of reflection from the principle that light should travel the shortest distance from the eye to the object. From the law of reflection, they deduced the images formed by plane and curved mirrors.

This is difficult stuff, and requires much training and study to learn. Sagan says the same about modern science at several points in the book, but in one chapter he vehemently contests the view that science is not part and parcel of human nature. Science is a natural proclivity, he asserts, that "is embedded deeply within us". Science is not hard to teach because it is hard, but because "indifference, inattention, incompetence, or fear of scepticism... discourage children from science". Why there is so much incompetency and fear of a natural proclivity is not explained, although elsewhere he admits that seven-year-olds are much better at wonder than scepticism.

The innateness of science is a deeply held Sagan belief. He denies that science had a unique origin in ancient Greece, claiming that the tracking know-how of preliterate peoples is science comparable to that of the Greeks. This claim goes beyond rejecting the theoretical accomplishments of the Greeks, to rejecting his own views of science given elsewhere in the book. If tracking know-how is science, why not make the same claim for a chimpanzee's ability to collect termites with a grass reed? Amazingly, Sagan does, defending his belief as uncritically as any alien abductee.

Sagan's passionate advocacy for science and scepticism uneasily cohabits with his passionate advocacy of shaky scientific, political, social and moral posi- tions. The result is a book with an abundance of material on which an aspiring sceptic can practise.

Alan Cromer is in the Department of Physics, Northeastern University, Boston, Massachusetts 02115, USA.

\section{Regal recipes}

\section{John Mann}

Classics in Total Synthesis: Targets, Strategies, Methods. By K. C. Nicolaou and E. J. Sorenson. VCH: 1996. Pp. 798. DM128, \$80, £52 (hbk); DM78, \$49.95, $£ 32$ (pbk).

IT is not often that a chemical structure graces the front cover of Nature, but such was the honour accorded to the complex natural product taxol* on 17 February 1994. It advertised the publication (in that issue - Nature 367, 630; 1994) of a paper recording an elegant total synthesis of taxol by K. C. Nicolaou and colleagues. The molecule had provided a formidable challenge (and still does) with its 11 stereocentres and dense array of functionality, yet the synthetic pathway reported was intriguingly simple, stereoselective and at least partially convergent. In short, a classic total synthesis and one of the 36 such syntheses gathered together by Nicolaou and Sorenson in this book.

But what constitutes a classic total synthesis? The authors have restricted themselves to natural products rather than theoretically interesting molecules such as cubane or dodecahedrane, or man-made structures such as dendrimers or spherands. So most of the examples demonstrate the great artistry and ingenuity of synthetic chemists as they struggled to assemble ever more complex natural products during the past 40 years. Much of this artistry depended on the invention of new reactions or methods for the control of stereochemistry, and perhaps most importantly on the ability to analyse the problem before starting the syntheses. E. J. Corey's exposition of the rules of retrosynthetic analysis in the late 1960s revolutionized the way chemists plan a synthesis, and his book The Logic of Chemical Synthesis (Wiley, 1989) summarized the methodology and reviewed his own 'classic' syntheses.

Each of the 36 chapters in Nicolaou and Sorenson's book spotlights one natural product and reveals the intimate details of its synthesis. Woodward's route to strychnine is not unexpectedly in 'poll position', not least because of its ingenuity and use of none but the simplest of chemical reagents. Although completed in 1954,

* Bristol-Myers Squibb has registered Taxol as a trade mark and wishes the scientific community to use the name paclitaxel. it remained the only total synthesis of this complex molecule until the newer routes of Magnus, Overman and others appeared in the early 1990 s. It was also important for demonstrating that a complex molecule could succumb to total synthesis, and so encouraged others to take the plunge. Woodward was also involved, with Eschenmoser, in the 12-year international effort that culminated in the synthesis of vitamin B12 in 1973. Like so many of the syntheses detailed in the book, the invention of new enabling chemistry along the way was just as important as the final triumph, and these novel reactions are highlighted throughout the text.

Not all of the molecular targets are complex, and the synthesis of the simple monoterpene menthol by Takasago and colleagues is used to exemplify important advances in catalytic asymmetric reactions. The preparation of the simple hexoses using Masamune-Sharpless asymmetric epoxidation and related techniques provides a further insight into the power of these new techniques. But it is the big molecules (the 'synthetic Everests') that take pride of place. Synthesis of the antineoplastic macrolide cytovaricin provides an opportunity to explore the use of the Evans chiral auxiliaries in asymmetric synthesis; and the preparation of the immunosuppressant rapamycin involves an examination of the palladium-catalysed reactions introduced by Heck, Stille and Suzuki. The current vogue for tandem radical processes is brought to the fore in the chapter on hirsutene synthesis, and 18 other syntheses that use this strategy are also discussed. The last two chapters reveal the enormous complexity of marine natural products and chronicle the triumphs of Kishi's and Nicolaou's groups in their syntheses of palytoxin and brevetoxin B.

Along with all this methodology, each chapter includes essential information about the occurrence and biological significance of the natural products, a full retrosynthetic analysis of the target structure and a detailed account of the actual synthesis. Mechanisms of the reactions and problems encountered are also examined. The quality of the illustrations is superb and there is a comprehensive list of references in each chapter. The book is not only a record of synthetic achievement during the past 40 years, but also a valuable research and teaching aid.

Not everyone will agree with the authors' choice of 'classics'. The few European contributions, for instance, are largely buried within the various chapters. But there is no denying that this superb book about the art and science of natural product synthesis will be an essential purchase for many organic chemists.

John Mann is in the Department of Chemistry, University of Reading, Reading RG6 6AD, UK.

NATURE - VOL $380 \cdot 28$ MARCH 1996 